\title{
Bidding in a Possibly Common-Value Auction*
}

\author{
Yuk-fai Fong $^{a, \dagger}$ and Daniel F. Garrett ${ }^{\mathrm{b}}$ \\ ${ }^{a}$ Management and Strategy Department, Northwestern University, 2001 Sheridan Rd., \\ Evanston, IL 60208 \\ ${ }^{\mathrm{b}}$ Department of Economics, Northwestern University, 2001 Sheridan Rd., Evanston, \\ IL 60208
}

\begin{abstract}
We analyze a second-price auction with two bidders in which only one of the bidders is informed as to whether the object is valued commonly. We show that any equilibrium strategy of the bidder who is uninformed must be part of an equilibrium when both bidders instead know that the auction is not common value, regardless of the way in which the values are different. We derive sufficient conditions for equilibrium existence.
\end{abstract}

JEL classification: D44, D82

Keywords: second-price auction; common value; model uncertainty

*We would like to thank George Deltas and Asher Wolinsky for useful comments and suggestions. The paper has significantly benefited from the help of an anonymous referee and an anonymous advisory editor. We also thank participants of the 2008 Summer Workshop in Industrial Organization and Management Strategy and Fall 2008 Midwest Mathematical Economics and Theory conference. Any errors are those of the authors.

${ }^{\dagger}$ Corresponding author. Fax: 18474671777

Email addresses: y-fong@kellogg.northwestern.edu (Y-f. Fong), dfgarrett@gmail.com (D.F. Garrett). 


\section{Introduction}

The popular press seems to be intrigued by the uncertainty about the motivations and identities of bidders in auctions and takes particular interest when a high-stakes auction has been won by a "mystery bidder". ${ }^{1}$ However, to a dealer who seeks resale profit, the presence of a mystery bidder poses a possible challenge for formulating a bidding strategy. If both the dealer and his opponent compete in the same downstream market, then they might well have the same value for an artwork after revelation of all privately held information. $^{2}$ On the other hand, if his opponent is a private collector, their valuations are likely to be much less interdependent. It is therefore natural to ask how a dealer would bid against a mystery bidder who may be either a dealer or a collector.

This paper studies an auction that is motivated by such a situation. ${ }^{3}$ We consider an auction (the Mixed Game) in which two bidders play one of two games: Game $C$, a second-price common-value auction, or Game $N$, a second-price auction where values are not common. One bidder (Bidder 2) knows which game is being played (she is modelinformed), but the other (Bidder 1) does not (he is model-uninformed). In Game N, we allow for an arbitrary relationship between valuations - this includes the possibility of private values as well as intermediate dependence (i.e., "interdependent values"). We restrict attention to equilibria in bid functions that satisfy a certain smoothness property.

\footnotetext{
${ }^{1}$ Among the numerous reports about mystery bidders, one example is a New York Times article 'Recognize this man? The art world doesn't' (May 6, 2006) that reported that an unidentified man bid $\$ 95$ million for the Picasso "Dora Maar with Cat". This was a close second to the highest price paid for an artwork at auction. As another example, a New York Times article 'Rothko Breaks a Record for Contemporary Art' (May 16, 2007) discusses the sale of paintings to three separate mystery bidders at the prices of $\$ 72.8$, $\$ 52.6$ and $\$ 14.6$ million.

${ }^{2}$ Several authors have used dealers bidding on artwork to exemplify common-value auctions. Examples include Deltas and Engelbrecht-Wiggans (2005) and Harrington (2009; chapter 10.4, p 301).

${ }^{3}$ Such situations are not exclusive to art auctions. Mystery bidders are also present in other settings that might otherwise involve common values such as corporate takeovers. For instance, when Pepsi recently acquired the majority stake in Russia's biggest juicemaker OAO Lebedyansky for $\$ 1.4$ billion, it submitted the bid without revealing its identity (see 'Lebedyansky Shares Gain on Speculation Pepsi May Bid (Update1)', Bloomberg.com, March 11, 2008, and 'Pepsi to buy into Russian juice maker for $\$ 1.4$ bln' uk.reuters.com, March 20, 2008).
} 
Given this restriction, we show that, in any equilibrium of the Mixed Game, the bid functions used in Game N constitute equilibrium strategies in Game N.

This follows from a peculiar feature of second-price common-value auctions (see Milgrom, 1981): essentially any strategy for Bidder 1, paired with Bidder 2's best response to it, constitutes an equilibrium of Game C. Now, consider any equilibrium of the Mixed Game. By virtue of Bidder 2's best response and the above property of common-value auctions, Bidder 1's strategy must be a best response in Game C. The key step is to show that Bidder 1 must also be playing a best response in Game N. Suppose he does not. Then a small deviation towards the Game $\mathrm{N}$ best response would yield him a first-order gain in Game N. It would yield him only a second-order loss in Game C, since the putative equilibrium strategy constitutes a best response in Game C. He would therefore want to deviate.

We thus conclude that the bidders coordinate with reference to the non-common-values equilibrium. Using this property, we derive sufficient conditions for equilibrium existence.

Our paper relates to several papers that have investigated the sensitivity of equilibria to small differences in values or small amounts of private information in otherwise commonvalue environments. Notable examples include Bikhchandani (1988), Bulow et al. (1999), Klemperer (1998), and Larson (2009). ${ }^{4}$ Bikhchandani's paper is the closest to our work. He analyzes a particular case of our model whereby, in Game N, the model-informed Bidder 2 has a higher valuation than her opponent with certainty. He shows, among other things, that Bidder 2 wins with certainty in case Game $\mathrm{N}$ occurs.

\footnotetext{
${ }^{4}$ In a similar spirit, Parreiras (2006) shows that a small probability that a second-price auction is in fact a first-price auction eliminates the multiplicity of equilibria associated with second-price common-value auctions.
} 


\section{Model}

Consider a second-price auction with two bidders. ${ }^{5}$ There are two possible states of the world: $\mathrm{C}$ and $\mathrm{N}$. It is common knowledge that state $\mathrm{C}$ occurs with probability $\theta$ and that state $\mathrm{N}$ occurs with probability $1-\theta$. In state $\mathrm{C}$, Bidder 1 (he) and Bidder 2 (she) have a common value for the object. In state $\mathrm{N}$, they may not. Bidder 2 alone observes the state. We refer to the situation in which $\theta \in(0,1)$ as the Mixed Game. If $\theta=1$ we call it Game $C$ and if $\theta=0$ we call it Game $N$.

Each bidder $i \in\{1,2\}$ observes a private signal $s_{i}$, distributed independently of the state. Conditional on the opponent's signal $s_{j}$, bidder $i$ 's signal has a density $f_{i}\left(\cdot \mid s_{j}\right)$ that is strictly positive on $[0,1]$. For each bidder $i, f_{i}(\cdot \mid \cdot)$ is continuous in both arguments. Assume the signals satisfy the monotone likelihood ratio property (MLRP). That is, for any $s_{i}^{\prime} \geq s_{i}$ and $s_{j}^{\prime} \geq s_{j}$

$$
\frac{f_{i}\left(s_{i}^{\prime} \mid s_{j}^{\prime}\right)}{f_{i}\left(s_{i} \mid s_{j}^{\prime}\right)} \geq \frac{f_{i}\left(s_{i}^{\prime} \mid s_{j}\right)}{f_{i}\left(s_{i} \mid s_{j}\right)}
$$

In state $\mathrm{C}$, both bidders have the same valuation $v:[0,1]^{2} \rightarrow \mathbb{R}$ that is increasing in both arguments. In state $\mathrm{N}$, each bidder $i$ has a valuation $v_{i N}:[0,1]^{2} \rightarrow \mathbb{R}$ that is strictly increasing in his or her own signal and non-decreasing in the opponent's. All values $v, v_{1 N}$ and $v_{2 N}$ are continuously differentiable in both arguments.

A strategy for Bidder 2 in the Mixed Game is a pair of bid functions $\left(\beta_{2 C}, \beta_{2 N}\right)$ that map signals to bids in each state. A strategy for Bidder 1 is simply $\beta_{1}$, as he does not observe the state. We consider interim Bayesian Nash equilibria and restrict attention to undominated strategies of the Mixed Game - that is, equilibria in which none of the strategies are weakly dominated. We also restrict attention to strictly increasing bid functions that are continuous and that are continuously differentiable at all but finitely

\footnotetext{
${ }^{5}$ Because there are only two bidders, the English button auction and the Vickrey auction are strategically equivalent.
} 
many points. ${ }^{6}$ Define the bid intervals $B_{1}=\left[\beta_{1}(0), \beta_{1}(1)\right], B_{2 C}=\left[\beta_{2 C}(0), \beta_{2 C}(1)\right]$ and $B_{2 N}=\left[\beta_{2 N}(0), \beta_{2 N}(1)\right]$. Each bid function can be represented equivalently by its inverse $-\sigma_{1}, \sigma_{2 C}$ and $\sigma_{2 N}$ respectively.

\section{Characterization of equilibrium}

A well-known condition for equilibrium, when applied to the Mixed Game, requires that Bidder 1 bid his value conditional on tying with his opponent, whenever ties are possible. This value depends on two dimensions of his opponent's information - her signal and the state. If Bidder 2 bids $p \in B_{2 C} \backslash B_{2 N}$, then Bidder 1 must infer state C. If she bids $p \in B_{2 N} \backslash B_{2 C}$, he must infer state N. When $p \in B_{2 C} \cap B_{2 N}$, his inference must be derived according to Bayes' Law.

Let $D$ be the set of points $p \in B_{2 C} \cap B_{2 N}$ at which both $\sigma_{2 C}$ and $\sigma_{2 N}$ are continuously differentiable. When Bidder 1 has signal $s_{1}$ and observes bidder 2 bid $p \in D$, the probability he assigns to state $\mathrm{C}$ is

$$
\hat{\theta}\left(s_{1}, p\right)=\frac{\theta f_{2}\left(\sigma_{2 C}(p) \mid s_{1}\right) \sigma_{2 C}^{\prime}(p)}{\theta f_{2}\left(\sigma_{2 C}(p) \mid s_{1}\right) \sigma_{2 C}^{\prime}(p)+(1-\theta) f_{2}\left(\sigma_{2 N}(p) \mid s_{1}\right) \sigma_{2 N}^{\prime}(p)} .
$$

Bidder 1's updating rule is thus defined for Bidder 2's bids in the set $U=D \cup\left(B_{2 C} \backslash B_{2 N}\right) \cup\left(B_{2 N} \backslash B_{2 C}\right)$. Bidder 1's expected valuation conditional on receiving signal $s_{1}$ and observing opponent bid $p \in U$ is

$$
w\left(s_{1}, p\right)= \begin{cases}\hat{\theta}\left(s_{1}, p\right) v\left(s_{1}, \sigma_{2 C}(p)\right)+\left(1-\hat{\theta}\left(s_{1}, p\right)\right) v_{1 N}\left(s_{1}, \sigma_{2 N}(p)\right) & \text { if } p \in D, \\ v\left(s_{1}, \sigma_{2 C}(p)\right) & \text { if } p \in B_{2 C} \backslash B_{2 N} \\ v_{1 N}\left(s_{1}, \sigma_{2 N}(p)\right) & \text { if } p \in B_{2 N} \backslash B_{2 C} .\end{cases}
$$

We can now state our central result.

\footnotetext{
${ }^{6}$ We briefly discuss the possibility of equilibria in discontinuous bid functions at the end of Section 4.
} 
Proposition 1 If $\left(\beta_{1},\left(\beta_{2 C}, \beta_{2 N}\right)\right)$ is an equilibrium in undominated strategies in the Mixed Game for any probability of state $C$, then $\left(\beta_{1}, \beta_{2 N}\right)$ is an equilibrium (possibly in dominated strategies) of Game $N$.

Proof approach. Suppose that $\left(\beta_{1},\left(\beta_{2 C}, \beta_{2 N}\right)\right)$ is an equilibrium in the Mixed Game. Since $\beta_{2 N}$ must be a best response to $\beta_{1}$, we need only verify that $\beta_{1}$ is a best response to $\beta_{2 N}$. Lemma 1 gives necessary conditions for $\beta_{1}$ to be a best response to $\left(\beta_{2 C}, \beta_{2 N}\right)$ in the Mixed Game. Lemma 2 gives a necessary condition for $\beta_{2 C}$ to be a best response to $\beta_{1}$. Together, these imply certain conditions (given in Lemma 3 ) that imply $\beta_{1}$ is a Game-N best response to $\beta_{2 N}$ (as shown in Lemma 4).

Lemma 1 For any $\theta \in(0,1)$, Bidder 1 is best responding to Bidder 2 only if

(i) for all $p \in B_{1} \cap U, w\left(\sigma_{1}(p), p\right)=p$;

(ii) for all $p<\beta_{1}(0)$,

$$
\begin{aligned}
\theta \int_{\left\{\widetilde{s}_{2}: p<\beta_{2 C}\left(\widetilde{s}_{2}\right)<\beta_{1}(0)\right\}} & \left(v\left(0, \widetilde{s}_{2}\right)-\beta_{2 C}\left(\widetilde{s}_{2}\right)\right) f_{2}\left(\widetilde{s}_{2} \mid 0\right) d \widetilde{s}_{2} \\
& +(1-\theta) \int_{\left\{\widetilde{s}_{2}: p<\beta_{2 N}\left(\widetilde{s}_{2}\right)<\beta_{1}(0)\right\}}\left(v_{1 N}\left(0, \widetilde{s}_{2}\right)-\beta_{2 N}\left(\widetilde{s}_{2}\right)\right) f_{2}\left(\widetilde{s}_{2} \mid 0\right) d \widetilde{s}_{2} \geq 0 ; \text { and }
\end{aligned}
$$

(iii) for all $p>\beta_{1}(1)$

$$
\begin{aligned}
\theta \int_{\left\{\widetilde{s}_{2}: \beta_{1}(1)<\beta_{2 C}\left(\widetilde{s}_{2}\right)<p\right\}} & \left(v\left(1, \widetilde{s}_{2}\right)-\beta_{2 C}\left(\widetilde{s}_{2}\right)\right) f_{2}\left(\widetilde{s}_{2} \mid 1\right) d \widetilde{s}_{2} \\
& +(1-\theta) \int_{\left\{\widetilde{s}_{2}: \beta_{1}(1)<\beta_{2 N}\left(\widetilde{s}_{2}\right)<p\right\}}\left(v_{1 N}\left(1, \widetilde{s}_{2}\right)-\beta_{2 N}\left(\widetilde{s}_{2}\right)\right) f_{2}\left(\widetilde{s}_{2} \mid 1\right) d \widetilde{s}_{2} \leq 0 .
\end{aligned}
$$

Proof. Conditions (ii) and (iii) simply state that Bidder 1 does not want to deviate downwards when $s_{1}=0$, nor upwards when $s_{1}=1$. Condition (i) states that, at any bid $p$ in the range of possible bids for both bidders, Bidder 1 is bidding his expected value conditional on receiving signal $\sigma_{1}(p)$ and tying with Bidder 2, provided the expectation 
is well-defined. This requirement follows from standard arguments (see, e.g., Milgrom (1981))

Lemma 2 Bid function $\beta_{2 C}$ is a best response for Bidder 2 to $\beta_{1}$ in state $C$ only if $v\left(\sigma_{1}(p), \sigma_{2 C}(p)\right)=p$ for every $p \in B_{1} \cap B_{2 C}$.

Again the proof is standard, and hence omitted.

Lemma 3 If $\left(\beta_{1},\left(\beta_{2 C}, \beta_{2 N}\right)\right)$ is an equilibrium in undominated strategies in the Mixed Game, then

(i) for all $p \in B_{1} \cap B_{2 N}, v_{1 N}\left(\sigma_{1}(p), \sigma_{2 N}(p)\right)=p$;

(ii) for all $p<\beta_{1}(0), \int_{\left\{\widetilde{s}_{2}: p<\beta_{2 N}\left(\widetilde{s}_{2}\right)<\beta_{1}(0)\right\}}\left(v_{1 N}\left(0, \widetilde{s}_{2}\right)-\beta_{2 N}\left(\widetilde{s}_{2}\right)\right) f_{2}\left(\widetilde{s}_{2} \mid 0\right) d \widetilde{s}_{2} \geq 0$; and

(iii) for all $p>\beta_{1}(1), \int_{\left\{\widetilde{s}_{2}: \beta_{1}(1)<\beta_{2 N}\left(\widetilde{s}_{2}\right)<p\right\}}\left(v_{1 N}\left(1, \widetilde{s}_{2}\right)-\beta_{2 N}\left(\widetilde{s}_{2}\right)\right) f_{2}\left(\widetilde{s}_{2} \mid 1\right) d \widetilde{s}_{2} \leq 0$.

Proof. For Bidder 2's strategy to be undominated in the Mixed Game requires that, for any $s_{2} \in[0,1], v\left(0, s_{2}\right) \leq \beta_{2 C}\left(s_{2}\right) \leq v\left(1, s_{2}\right)$. Clearly, then, the first integral in Lemma 1(ii) is non-positive and the first integral in Lemma 1(iii) is non-negative. Conditions (ii) and (iii) of this lemma follow.

For (i), let $p \in B_{1} \cap B_{2 N}$. If $p \notin B_{2 C}$, it holds by Lemma 1(i). If $p \in B_{2 C} \cap D$, then it follows by subtracting $v\left(\sigma_{1}(p), \sigma_{2 C}(p)\right)=p$ (in Lemma 2) from the equation in Lemma 1(i). Remaining possible values for $p$ are in $\left(B_{2 C} \cap B_{2 N}\right) \backslash D$, which is at most finite. Condition (i) therefore follows by continuity of $v_{1 N}\left(\sigma_{1}(\cdot), \sigma_{2 N}(\cdot)\right)$ on $B_{1} \cap B_{2 N}$.

Lemma 4 If bid functions $\beta_{1}$ and $\beta_{2 N}$ satisfy conditions (i), (ii) and (iii) of Lemma 3, then $\beta_{1}$ is a best response for Bidder 1 to $\beta_{2 N}$ when he knows the state is $N$.

Proof. We consider only downward deviations, as upward deviations can be ruled out analogously. Let $s_{1} \in[0,1]$. By standard arguments (e.g., Milgrom, 1981), Lemma 1(i) guarantees that any deviation to a bid $\hat{b} \in\left[\beta_{1}(0), \beta_{1}\left(s_{1}\right)\right)$ is unprofitable. Consider, then, a deviation to $\hat{b}<\beta_{1}(0)$. This is unprofitable if 


$$
\int_{\left\{\widetilde{s}_{2}: \hat{b}<\beta_{2 N}\left(\widetilde{s}_{2}\right)<\beta_{1}\left(s_{1}\right)\right\}}\left(v_{1 N}\left(s_{1}, \widetilde{s}_{2}\right)-\beta_{2 N}\left(\widetilde{s}_{2}\right)\right) f_{2}\left(\widetilde{s}_{2} \mid s_{1}\right) d \widetilde{s}_{2} \geq 0 .
$$

The unprofitability of deviation $\hat{b}=\beta_{1}(0)$ implies that the above inequality holds for integration over the set $\left\{\widetilde{s}_{2}: \beta_{1}(0) \leq \beta_{2 N}\left(\widetilde{s}_{2}\right)<\beta_{1}\left(s_{1}\right)\right\}$. Therefore, it is enough to show it holds when integration is over only $\left\{\widetilde{s}_{2}: \hat{b}<\beta_{2 N}\left(\widetilde{s}_{2}\right)<\beta_{1}(0)\right\}$. Since $v_{1 N}$ is increasing in $s_{1}$, it is enough to show that

$$
\int_{\left\{\widetilde{s}_{2}: \hat{b}<\beta_{2 N}\left(\widetilde{s}_{2}\right)<\beta_{1}(0)\right\}}\left(v_{1 N}\left(0, \widetilde{s}_{2}\right)-\beta_{2 N}\left(\widetilde{s}_{2}\right)\right) f_{2}\left(\widetilde{s}_{2} \mid s_{1}\right) d \widetilde{s}_{2} \geq 0 .
$$

This is immediate from Lemma 3(ii) when the bidders' signals are independent. Otherwise, an argument using the MLRP is needed, and this is given in the Appendix.

Consider now the Mixed Game with some fixed probability of state $\mathrm{C}, \theta \in(0,1)$. It is interesting to ask whether an equilibrium $\left(\beta_{1},\left(\beta_{2 C}, \beta_{2 N}\right)\right)$ of the Mixed Game for the probability $\theta$ must also be an equilibrium for other values of this probability. Since $\left(\beta_{1}, \beta_{2 N}\right)$ is an equilibrium in Game $\mathrm{N}$, it is easy to show that the equilibrium persists for probabilities lower than $\theta$. However, for higher probabilities (including probability 1 - i.e., in Game C), this need not be the case. Suppose that there exist signals $s_{2}$ such that Bidder 2 wins with certainty in state $\mathrm{C}$, and that for such signals $\beta_{2 C}\left(s_{2}\right)<v\left(1, s_{2}\right)$. Although Bidder 1 , when receiving signal $s_{1}=1$, would not wish to deviate for low probabilities of state $\mathrm{C}$ (because it would be detrimental in case state $\mathrm{N}$ occurs), he may well wish to deviate upwards for high probabilities. To ensure this is not the case, we might impose a further restriction on Bidder 2's possible strategies in the Mixed Game. ${ }^{7}$

$\mathbf{R S}(\mathbf{C})$ : Bidder 2's strategy satisfies $\beta_{2 C}\left(s_{2}\right)=v\left(1, s_{2}\right)$ whenever $\beta_{2 C}\left(s_{2}\right)>\beta_{1}(1)$ and $\beta_{2 C}\left(s_{2}\right)=v\left(0, s_{2}\right)$ whenever $\beta_{2 C}\left(s_{2}\right)<\beta_{1}(0)$.

The next result then follows from the arguments above.

\footnotetext{
${ }^{7}$ This condition is implicitly assumed in Proposition 2 of Bikhchandani's (1988) paper.
} 
Proposition 2 Suppose the strategy pair $\left(\beta_{1},\left(\beta_{2 C}, \beta_{2 N}\right)\right)$ is undominated in the Mixed Game and $\beta_{2 C}$ satisfies $R S(C)$. It is an equilibrium in the Mixed Game if and only if $\left(\beta_{1}, \beta_{2 C}\right)$ is an equilibrium in Game $C$ and $\left(\beta_{1}, \beta_{2 N}\right)$ is an equilibrium in Game $N$.

\section{Existence of equilibrium}

Suppose we confine our interest to equilibria satisfying $\mathrm{RS}(\mathrm{C})$. Then, by Proposition 2, the question of existence may be answered by considering whether there are functions $\beta_{1}, \beta_{2 C}$ and $\beta_{2 N}$ such that $\left(\beta_{1}, \beta_{2 C}\right)$ is an equilibrium of Game $\mathrm{C}$ and $\left(\beta_{1}, \beta_{2 N}\right)$ is an equilibrium of Game N. We give conditions for equilibrium existence in Proposition 3 below.

We find it convenient to consider equilibrium bid functions $\beta_{1}$ and $\beta_{2 N}$ that satisfy analogues of the condition $\mathrm{RS}(\mathrm{C})$.

$\mathbf{R S}(\mathbf{N})$ : Bidder 2's strategy satisfies $\beta_{2 N}\left(s_{2}\right)=v_{2 N}\left(1, s_{2}\right)$ whenever $\beta_{2 N}\left(s_{2}\right)>\beta_{1}(1)$ and $\beta_{2 N}\left(s_{2}\right)=v_{2 N}\left(0, s_{2}\right)$ whenever $\beta_{2 N}\left(s_{2}\right)<\beta_{1}(0)$.

RS(1): Bidder 1's strategy satisfies $\beta_{1}\left(s_{1}\right)=v_{1 N}\left(s_{1}, 1\right)$ whenever $\beta_{1}\left(s_{1}\right)>\beta_{2 N}(1)$ and $\beta_{1}\left(s_{1}\right)=v_{1 N}\left(s_{1}, 0\right)$ whenever $\beta_{1}\left(s_{1}\right)<\beta_{2 N}(0)$.

Proposition 3 There exists an equilibrium of the Mixed Game in undominated strategies that satisfies $R S(1), R S(C)$ and $R S(N)$ if, for all $s_{1}, s_{2 C}, s_{2 N} \in[0,1], 8$

$$
\begin{aligned}
& \frac{\partial v_{1 N}\left(s_{1}, s_{2 N}\right)}{\partial s_{1}}>\frac{\partial v_{2 N}\left(s_{1}, s_{2 N}\right)}{\partial s_{1}} \text { and } \frac{\partial v_{2 N}\left(s_{1}, s_{2 N}\right)}{\partial s_{2}}>\frac{\partial v_{1 N}\left(s_{1}, s_{2 N}\right)}{\partial s_{2}} \\
& \frac{\partial v_{1 N}\left(s_{1}, s_{2 N}\right)}{\partial s_{1}}>\frac{\partial v\left(s_{1}, s_{2 C}\right)}{\partial s_{1}} ; \text { and } \\
& \frac{\partial v_{1 N}\left(s_{1}, s_{2 N}\right)}{\partial s_{1}} \frac{\partial v_{2 N}\left(s_{1}, s_{2 N}\right)}{\partial s_{2}}-\frac{\partial v_{2 N}\left(s_{1}, s_{2 N}\right)}{\partial s_{1}} \frac{\partial v_{1 N}\left(s_{1}, s_{2 N}\right)}{\partial s_{2}}>\frac{\partial v\left(s_{1}, s_{2 C}\right)}{\partial s_{1}}\left(\frac{\partial v_{2 N}\left(s_{1}, s_{2 N}\right)}{\partial s_{2}}-\frac{\partial v_{1 N}\left(s_{1}, s_{2 N}\right)}{\partial s_{2}}\right) .
\end{aligned}
$$

Condition (i) is the "single-crossing condition" suggested initially by Maskin (1992). It guarantees existence of an equilibrium in Game N. Condition (ii) says that Bidder 1's

\footnotetext{
${ }^{8}$ Conditions (i)-(iii) regulate the slopes of the bid functions via the conditions for equilibrium that we outline in the proof below. We must consider Bidder 2's bid functions for both state $\mathrm{C}$ and N. However, at any given bid $p$, Bidder 2's state $\mathrm{C}$ and state $\mathrm{N}$ signals $\left(\sigma_{2 C}(p)\right.$ and $\left.\sigma_{2 N}(p)\right)$ may differ. To account for this possibility, we impose the conditions for possibly distinct values $s_{2 C}$ and $s_{2 N}$.
} 
signal has a greater marginal effect on his value when the state is $\mathrm{N}$ than on the common value. Condition (iii) is more difficult to interpret, but is implied by conditions (i) and (ii) together with the requirement that, for all $s_{1}, s_{2 C}, s_{2 N} \in[0,1]$,

$$
\frac{\partial v\left(s_{1}, s_{2 C}\right)}{\partial s_{1}} \geq \frac{\partial v_{2 N}\left(s_{1}, s_{2 N}\right)}{\partial s_{1}}
$$

Proof of Proposition 3. Given condition (i), arguments similar to Lemma 2 of Krishna (2003) imply that an equilibrium in undominated strategies exists in Game N, with bid functions satisfying $\mathrm{RS}(1)$ and $\mathrm{RS}(\mathrm{N})$. Suppose that $\left(\beta_{1}, \beta_{2 N}\right)$ is such an equilibrium. We construct a best response $\beta_{2 C}$ to $\beta_{1}$ that satisfies $\mathrm{RS}(\mathrm{C})$. It follows immediately that $\beta_{1}$ is a best response to $\beta_{2 C}$ in state $\mathrm{C}$, establishing that $\left(\beta_{1},\left(\beta_{2 C}, \beta_{2 N}\right)\right)$ is an equilibrium.

The construction of Bidder 2's best response $\beta_{2 C}$ proceeds in three steps. Step 1 shows that $\beta_{1}$ is "sufficiently steep" to admit a continuous and increasing best response by Bidder 2 in state C. Step 2 defines the interval over which ties can occur in state C, and Step 3 constructs $\beta_{2 C}$ as the solution to a differential equation. Verifying that $\beta_{2 C}$ is a best response is left to the reader.

Step 1. In this step, we show that, for any $p \in B_{1}$ and any $s_{2 C} \in[0,1]$, and provided that $\sigma_{1}$ is differentiable at $p$,

$$
\frac{\partial v\left(\sigma_{1}(p), s_{2 C}\right)}{\partial s_{1}} \frac{d \sigma_{1}(p)}{d p}<1
$$

To see this, note that, if either $p<\beta_{2 N}(0)$ so that (by $\left.\operatorname{RS}(1)\right) \sigma_{1}$ solves

$$
\frac{\partial v_{1 N}\left(\sigma_{1}(p), 0\right)}{\partial s_{1}} \frac{d \sigma_{1}(p)}{d p}=1
$$

or, if $p>\beta_{2 N}(1)$ so that (again, by $\left.\operatorname{RS}(1)\right) \sigma_{1}$ solves

$$
\frac{\partial v_{1 N}\left(\sigma_{1}(p), 1\right)}{\partial s_{1}} \frac{d \sigma_{1}(p)}{d p}=1
$$

then the result follows by condition (ii). If $p \in B_{2 N}$, it is ensured by condition (iii). This 
is because the equilibrium requirement in Game $\mathrm{N}$ is

$$
v_{1 N}\left(\sigma_{1}(p), \sigma_{2 N}(p)\right)=v_{2 N}\left(\sigma_{1}(p), \sigma_{2 N}(p)\right)=p
$$

which, after differentiating with respect to $p$ and solving for $\frac{d \sigma_{1}(p)}{d p}$, yields

$$
\frac{d \sigma_{1}(p)}{d p}=\frac{\frac{\partial v_{2 N}\left(\sigma_{1}(p), \sigma_{2 N}(p)\right)}{\partial s_{2}}-\frac{\partial v_{1 N}\left(\sigma_{1}(p), \sigma_{2 N}(p)\right)}{\partial s_{2}}}{\frac{\partial v_{1 N}\left(\sigma_{1}(p), \sigma_{2 N}(p)\right)}{\partial s_{1}} \frac{\partial v_{2 N}\left(\sigma_{1}(p), \sigma_{2 N}(p)\right)}{\partial s_{2}}-\frac{\partial v_{2 N}\left(\sigma_{1}(p), \sigma_{2 N}(p)\right)}{\partial s_{1}} \frac{\partial v_{1 N}\left(\sigma_{1}(p), \sigma_{2 N}(p)\right)}{\partial s_{2}}} .
$$

Step 2. If $v(1,0)>\beta_{1}(1)$ or $v(0,1)<\beta_{1}(0)$ then Bidder 2's strategy can be specified according to $\mathrm{RS}(\mathrm{C})$, with Bidder 2 either winning or losing with certainty for all signals. So suppose that $v(1,0) \leq \beta_{1}(1)$ and $v(0,1) \geq \beta_{1}(0)$. If $v(0,0) \leq \beta_{1}(0)$ put $\underline{p}=\beta_{1}(0)$. If $v(0,0)>\beta_{1}(0)$, then since $v(1,0) \leq \beta_{1}(1)$, and since $\beta_{1}$ is continuous, we may specify $\underline{p}$ such that $v\left(\sigma_{1}(\underline{p}), 0\right)=\underline{p}$. By Step 1, this is uniquely defined. Similarly, if $v(1,1) \geq \beta_{1}(1)$, put $\bar{p}=\beta_{1}(1)$. If $v(1,1)<\beta_{1}(1)$, define $\bar{p}$ as satisfying $v\left(\sigma_{1}(\bar{p}), 1\right)=\bar{p}$. We will construct a best response for Bidder 2 for which ties are possible for bids in $[\underline{p}, \bar{p}]$.

Step 3. On the interval $[\underline{p}, \bar{p}]$, bids must be determined by

$$
v\left(\sigma_{1}(p), \sigma_{2 C}(p)\right)=p .
$$

An increasing and continuous function $\sigma_{2 C}$ that satisfies this equation can be constructed on $[\underline{p}, \bar{p}]$ as a solution to

$$
\frac{\partial v\left(\sigma_{1}(p), \sigma_{2 C}(p)\right)}{\partial s_{1}} \frac{d \sigma_{1}(p)}{d p}+\frac{\partial v\left(\sigma_{1}(p), \sigma_{2 C}(p)\right)}{\partial s_{2}} \frac{d \sigma_{2 C}(p)}{d p}=1
$$

(wherever the derivative of $\sigma_{1}$ exists), with initial condition given by $\sigma_{2 C}(\underline{p})=\underline{s}_{2}$, where $v\left(0, \underline{s}_{2}\right)=\underline{p}$, if $v(0,0) \leq \beta_{1}(0)$, and by $\sigma_{2 C}(\underline{p})=0$ otherwise. This is possible because $\frac{\partial v\left(\sigma_{1}, \sigma_{2 C}\right)}{\partial s_{1}} \frac{d \sigma_{1}}{d p}<1$ by Step 1 , and because $\frac{\partial v\left(\sigma_{1}, \sigma_{2 C}\right)}{\partial s_{2}}>0$. The function $\sigma_{2 C}$ can then be extended to $p \notin[\underline{p}, \bar{p}]$ according to $\mathrm{RS}(\mathrm{C})$.

Proposition 3 provides sufficient conditions for equilibrium existence that are fairly easy to check. A natural case to consider is when state $\mathrm{N}$ is pure private values. In this 
case, condition (i) is automatically satisfied and conditions (ii) and (iii) are equivalent. So an equilibrium exists provided that condition (ii) is satisfied.

The conditions of Proposition 3 are, however, somewhat more restrictive than necessary for the existence of the equilibria that we consider: i.e., those in undominated strategies, with bid functions that are increasing, that satisfy the continuity and differentiability conditions given in the model set-up, and that satisfy $\mathrm{RS}(1), \mathrm{RS}(\mathrm{C})$ and $\mathrm{RS}(\mathrm{N})$. There are two reasons for this. Condition (i) is more than required for equilibrium in Game $\mathrm{N}$ (although, this assumption has been made routinely in the literature on efficiency of second-price auctions). Also, the requirement that conditions (i)-(iii) hold for all possible signal realizations is more than required. Of course, this restriction does not matter if values are linear in the signals (since the derivatives in conditions (i)-(iii) are then independent of bidder signals).

Although the conditions are not necessary for the existence of the equilibria that we focus on, the following example illustrates why, when condition (ii) fails, there may be no equilibrium in undominated strategies with continuous and strictly increasing bid functions. ${ }^{9}$

Example 1 Suppose that, for all $s_{1}, s_{2} \in[0,1], v\left(s_{1}, s_{2}\right)=s_{1}+s_{2}, v_{1 N}\left(s_{1}, s_{2}\right)=a+n s_{1}$ and $v_{2 N}\left(s_{1}, s_{2}\right)=a+n s_{2}$, where $n<1$ and $1-n<a<1$. Suppose that $\theta \in(0,1)$ and that bidder signals are independently distributed. Then there is no equilibrium in the Mixed Game in undominated strategies with continuous and strictly increasing bid functions.

Proof. Suppose with a view to contradiction that $\left(\beta_{1},\left(\beta_{2 C}, \beta_{2 N}\right)\right)$ is an equilibrium in increasing, continuous bid functions and undominated strategies. That Bidder 2 plays an undominated strategy requires $\beta_{2 N}\left(s_{2}\right)=a+n s_{2}$ for any $s_{2} \in[0,1]$. For Bidder 1 to be

\footnotetext{
${ }^{9}$ Notice that this is true in the example without restricting attention to equilibria satisfying $\operatorname{RS}(1)$, $\operatorname{RS}(\mathrm{C})$ and $\operatorname{RS}(\mathrm{N})$.
} 
playing an undominated strategy requires that, for any $s_{1} \in[0,1], \beta_{1}\left(s_{1}\right)=\hat{\theta}\left(s_{1}+\hat{s}_{2}\right)+$ $(1-\hat{\theta})\left(a+n s_{1}\right)$ for some $\hat{s}_{2}, \hat{\theta} \in[0,1]$. Therefore, since $\beta_{1}$ is continuous (and given the restrictions on $a$ and $n), B_{1} \cap B_{2 N}$ must contain $p=1$ and have a non-empty interior. Since $\left(\beta_{1},\left(\beta_{2 C}, \beta_{2 N}\right)\right)$ satisfies the conditions of Proposition $1,\left(\beta_{1}, \beta_{2 N}\right)$ must be an equilibrium in Game N. Therefore, $\sigma_{1}(p)=\frac{p-a}{n}$ for $p \in \operatorname{int}\left[B_{1} \cap B_{2 N}\right]$. Since $\sigma_{1}(1)=\frac{1-a}{n} \in(0,1)$, and since $\sigma_{1}$ is strictly increasing and continuous, $1 \in \operatorname{int}\left[B_{1} \cap B_{2 N}\right]$. That Bidder 2 best responds in state $\mathrm{C}$ then requires $\frac{p-a}{n}+\sigma_{2 C}(p)=p$ and thus $\sigma_{2 C}^{\prime}(p)=1-\frac{1}{n}<0$ in a neighbourhood of 1 . This violates the assumption of increasing bid functions.

Whilst Example 1 suggests why the conditions of Proposition 3 are important for equilibria in the class we consider to exist, note that other equilibria, especially those in discontinuous bid functions, may be possible. For instance, consider Example 1 and suppose that signals are uniformly distributed. Then, there exists an equilibrium in undominated strategies with discontinuous bid functions satisfying $\operatorname{RS}(1), \operatorname{RS}(\mathrm{C})$ and $\operatorname{RS}(\mathrm{N})$. Indeed, the bid functions $\beta_{1}\left(s_{1}\right)=a+n s_{1}, \beta_{2 N}\left(s_{2}\right)=a+n s_{2}$, and

$$
\beta_{2 C}\left(s_{2}\right)=\left\{\begin{array}{cl}
s_{2} & \text { if } s_{2} \leq a-\frac{1-n}{2} \\
1+s_{2} & \text { if } s_{2}>a-\frac{1-n}{2}
\end{array}\right.
$$

constitute such an equilibrium. ${ }^{10}$ Furthermore, $\left(\beta_{1}, \beta_{2 N}\right)$ constitutes an equilibrium of Game N. It is therefore possible that, even when there is no equilibrium in continuous bid functions and undominated strategies, the conclusion of Proposition 1 remains valid.

Another example of some interest is the model proposed by Deltas and EngelbrechtWiggans (2005), where Bidder 2 may be "naïve" in the sense that she misunderstands the importance of the winner's curse and fails to take into account the information revealed by Bidder 1's bid. ${ }^{11}$ After a normalization of their model, bidder values are given,

\footnotetext{
${ }^{10}$ We are grateful to the anonymous referee for pointing this out.

${ }^{11}$ Deltas and Engelbrecht-Wiggans introduce their model to study the question of the evolutionary performance of naïve bidders. They consider the case in which Bidder 2 is known to be naïve, but express
} 
for all $s_{1}, s_{2} \in[0,1]$, by $v_{1 N}\left(s_{1}, s_{2}\right)=v\left(s_{1}, s_{2}\right)=s_{1} s_{2}$, and $v_{2 N}\left(s_{1}, s_{2}\right)=\mathbb{E}\left[\tilde{s}_{1}\right] s_{2}$, where $\mathbb{E}\left[\tilde{s}_{1}\right]$ is the unconditional expectation of Bidder 1's signal. Assume again that signals are independently distributed. Different from Example 1, both conditions (i) and (ii) of Proposition 3 fail here, but there still exists an equilibrium of the Mixed Game in undominated strategies such that $\left(\beta_{1}, \beta_{2 N}\right)$ is an equilibrium of Game N. In particular, the following bid functions constitute an equilibrium in undominated strategies satisfying $\operatorname{RS}(1), \operatorname{RS}(\mathrm{C})$ and $\operatorname{RS}(\mathrm{N}): \beta_{2 N}\left(s_{2}\right)=\beta_{2 C}\left(s_{2}\right)=\mathbb{E}\left[\tilde{s}_{1}\right] s_{2}$ and

$$
\beta_{1}\left(s_{1}\right)=\left\{\begin{array}{cl}
0 & \text { if } s_{1} \leq \mathbb{E}\left[\tilde{s}_{1}\right] \\
s_{1} & \text { if } s_{1}>\mathbb{E}\left[\tilde{s}_{1}\right] .
\end{array}\right.
$$

Note that $\beta_{1}$ is the same bid function Bidder 1 uses in the case when it is commonly known that Bidder 2 is naïve.

\section{Concluding remarks}

This paper has considered an auction in which one bidder is uncertain as to the correct model of valuations, namely whether values are common. Our central result is that, in this situation, bidders coordinate on the non-common values equilibrium. The result is relevant to situations in which the motivation of one of the bidders at an auction is uncertain. An example is where the identity of a bidder is uncertain (i.e., when there is a "mystery bidder"). Our result holds in an environment that accommodates both a general form of interdependent values in case values are not common, as well as correlation of bidder signals (subject to the MLRP).

Our finding that the non-common values equilibrium is focal would not carry over to general situations in which both bidders face uncertainty about the commonality of values. ${ }^{12}$ Nor would it apply directly to a second-price auction with more than two bidders, an interest in the case where this naïvety is uncertain.

${ }^{12}$ Indeed, de Frutos and Pechlivanos (2006) show that Bikhchandani's (1988) results, which depend on 
only some of whom know whether values are common. Nonetheless, the two-bidder end game of an English auction with such bidders might take exactly the form analyzed here. ${ }^{13}$

\section{References}

[1] Bikhchandani, S. 1988. Reputation in Repeated Second-Price Auctions. J. Econ. Theory. $46,97-119$.

[2] Bulow, J., Huang, M., Klemperer, P., 1999. Toeholds and takeovers. J. Polit. Economy. 107, 427-454.

[3] Deltas, G., Engelbrecht-Wiggans, R. 2005. Naive Bidding. Manage. Sci. 51, 328-338.

[4] de Frutos, M.-A., Pechlivanos, L. 2006. Second-price common-value auctions under multidimensional uncertainty. Games Econ. Behav. 55, 43-71.

[5] Harrington, J., 2009. Games, Strategies and Decision Making. Worth Publishers.

[6] Klemperer, P., 1998. Auctions with almost common values: The 'wallet game' and its applications. Europ. Econ. Rev. 42, 757-769.

[7] Krishna, V. 2003. Asymmetric English auctions. J. Econ. Theory. 112, 261-288.

[8] Larson, N. 2009. Private value perturbations and informational advantage in common value auctions. Games Econ. Behav. 65, 430-460.

[9] Maskin, E. 1992. Auctions and privatization. In: Siebert, H. (Ed.), Privatization, Institut fur Weltwirtschaften der Universität Kiel, Kiel, 115-136.

[10] Milgrom, P., 1981. Rational expectations, information acquisition, and competitive bidding. Econometrica 49, 921-943.

similar logic to ours, do not carry over to such an environment.

${ }^{13}$ Larson (2009) makes a similar observation in the context of his two-bidder model. 
[11] Parreiras, S. 2006. Affiliated Common Value Auctions with Differential Information: The Two Bidder Case. Contrib. to Theoretical Econ. 6, Article 12.

\section{Appendix}

This Appendix completes the proof of Lemma 4 in the proof of Proposition 1. Specifically, it is shown that if the bidders' signals obey the MLRP, condition (ii) of Lemma 3 implies that for any $s_{1} \in[0,1]$ and $\widehat{b}<\beta_{1}(0)$

$$
\int_{\left\{\widetilde{s}_{2}: \widehat{b}<\beta_{2 N}\left(\widetilde{s}_{2}\right)<\beta_{1}(0)\right\}}\left(v_{1 N}\left(0, \widetilde{s}_{2}\right)-\beta_{2 N}\left(\widetilde{s}_{2}\right)\right) f_{2}\left(\widetilde{s}_{2} \mid s_{1}\right) d \widetilde{s}_{2} \geq 0 .
$$

Let $\psi(s) \equiv v_{1 N}(0, s)-\beta_{2 N}(s)$, let $N(b) \equiv\left\{s \in[0,1]: b<\beta_{2 N}(s)<\beta_{1}(0)\right.$ and $\psi(s)<0\}$ and let $P(b) \equiv\left\{s \in[0,1]: b<\beta_{2 N}(s)<\beta_{1}(0)\right.$ and $\left.\psi(s)>0\right\}$. If $N(\widehat{b})$ is empty, then we are done, so suppose $N(\widehat{b})$ is nonempty. The set $N(\widehat{b})$ is open in the interval $[0,1]$ since $\psi$ is continuous. It follows that $N(\widehat{b})$ can be expressed as a countable union of some collection of disjoint balls that are open in $[0,1]$. Call this collection $\Gamma$.

Condition (ii) of Lemma 3 implies that for $b \in\left[\widehat{b}, \beta_{1}(0)\right]$,

$$
\int_{P(b)} \psi\left(\widetilde{s}_{2}\right) f_{2}\left(\widetilde{s}_{2} \mid 0\right) d \widetilde{s}_{2}+\int_{N(b)} \psi\left(\widetilde{s}_{2}\right) f_{2}\left(\widetilde{s}_{2} \mid 0\right) d \widetilde{s}_{2} \geq 0
$$

Each integral is continuous in $b$. Therefore, for any $x \in\left[\widehat{b}, \beta_{1}(0)\right]$ there exists a number $\phi(x) \geq x$ such that

$$
\int_{P(\phi(x))} \psi\left(\widetilde{s}_{2}\right) f_{2}\left(\widetilde{s}_{2} \mid 0\right) d \widetilde{s}_{2}=-\int_{N(x)} \psi\left(\widetilde{s}_{2}\right) f_{2}\left(\widetilde{s}_{2} \mid 0\right) d \widetilde{s}_{2}
$$

Let $\gamma \in \Gamma$, and define $\bar{\gamma} \equiv \sup \gamma, \underline{\gamma} \equiv \inf \gamma$ and $m(\gamma) \equiv P(\phi(\underline{\gamma})) \backslash P(\phi(\bar{\gamma}))$. Then

$$
\int_{m(\gamma)} \psi\left(\widetilde{s}_{2}\right) f_{2}\left(\widetilde{s}_{2} \mid 0\right) d \widetilde{s}_{2}=-\int_{\gamma} \psi\left(\widetilde{s}_{2}\right) f_{2}\left(\widetilde{s}_{2} \mid 0\right) d \widetilde{s}_{2} .
$$

Define $l(\gamma)=\inf _{s \in \gamma} \frac{f_{2}(s \mid 0)}{f_{2}\left(s \mid s_{1}\right)}$. Then for any $s \in \gamma, l(\gamma) f_{2}\left(s \mid s_{1}\right) \leq f_{2}(s \mid 0)$. Substituting into the above equation yields 


$$
\int_{m(\gamma)} \psi\left(\widetilde{s}_{2}\right) \frac{f_{2}\left(\widetilde{s}_{2} \mid 0\right)}{l(\gamma)} d \widetilde{s}_{2} \geq-\int_{\gamma} \psi\left(\widetilde{s}_{2}\right) f_{2}\left(\widetilde{s}_{2} \mid s_{1}\right) d \widetilde{s}_{2}
$$

For $s^{\prime} \in \gamma$ and $s^{\prime \prime} \in m(\gamma)$ we have $s^{\prime \prime}>s^{\prime}$. The MLRP therefore implies $\frac{f_{2}\left(s^{\prime} \mid 0\right)}{f_{2}\left(s^{\prime} \mid s_{1}\right)} \geq \frac{f_{2}\left(s^{\prime \prime} \mid 0\right)}{f_{2}\left(s^{\prime \prime} \mid s_{1}\right)}$, so that $l(\gamma) \geq \frac{f_{2}\left(s^{\prime \prime} \mid 0\right)}{f_{2}\left(s^{\prime \prime} \mid s_{1}\right)}$, or, equivalently,

$$
f_{2}\left(s^{\prime \prime} \mid s_{1}\right) \geq \frac{f_{2}\left(s^{\prime \prime} \mid 0\right)}{l(\gamma)}
$$

Since $s^{\prime \prime}$ was chosen arbitrarily from $m(\gamma)$

$$
\int_{m(\gamma)} \psi\left(\widetilde{s}_{2}\right) f_{2}\left(\widetilde{s}_{2} \mid s_{1}\right) d \widetilde{s}_{2} \geq-\int_{\gamma} \psi\left(\widetilde{s}_{2}\right) f_{2}\left(\widetilde{s}_{2} \mid s_{1}\right) d \widetilde{s}_{2}
$$

Therefore, it follows that

$$
\begin{aligned}
\int_{\left\{\widetilde{s}_{2}: \widehat{b}<\beta_{2 N}\left(\widetilde{s}_{2}\right)<\beta_{1}(0)\right\}} \psi\left(\widetilde{s}_{2}\right) f_{2}\left(\widetilde{s}_{2} \mid s_{1}\right) d \widetilde{s}_{2} & =\int_{P(\widehat{b})} \psi\left(\widetilde{s}_{2}\right) f_{2}\left(\widetilde{s}_{2} \mid s_{1}\right) d \widetilde{s}_{2}+\int_{N(\widehat{b})} \psi\left(\widetilde{s}_{2}\right) f_{2}\left(\widetilde{s}_{2} \mid s_{1}\right) d \widetilde{s}_{2} \\
& \geq \sum_{\gamma \in \Gamma}\left(\int_{m(\gamma)} \psi\left(\widetilde{s}_{2}\right) f_{2}\left(\widetilde{s}_{2} \mid s_{1}\right) d \widetilde{s}_{2}+\int_{\gamma} \psi\left(\widetilde{s}_{2}\right) f_{2}\left(\widetilde{s}_{2} \mid s_{1}\right) d \widetilde{s}_{2}\right) \\
& \geq 0 .
\end{aligned}
$$

The first inequality follows because, by construction, $\bigcup\{\gamma \in \Gamma\}=N(\widehat{b})$, whereas $\bigcup\{m(\gamma): \gamma \in \Gamma\} \subset P(\widehat{b})$ (whilst $\psi$ is negative on $N(\widehat{b})$ and positive on $P(\widehat{b})$ ). The second inequality follows by $(*)$. 